\title{
Isolement et caractérisation de la fraction hydrophobe des protéose-peptones des laits bovin, ovin et caprin
}

\author{
A Mati ${ }^{1}$, JM Girardet ${ }^{1}$, D Xenakis $2^{*}$, G Linden 1 \\ ${ }^{1}$ Laboratoire de biochimie appliquée; associé à L'INRA, BP 239, \\ 54506 Vandouvre-les-Nancy Cedex, France; \\ 2 Université d'agriculture d'Athènes, laboratoire de technologie laitière, Grèce
}

(Reçu le 9 octobre 1990; accepté le 15 décembre 1990)

\begin{abstract}
Résumé - Le protocole d'isolement des protéose-peptones, largement utilisé jusque là pour le lait de vache, a été adapté et appliqué aux laits de brebis et de chèvre. Les fractions de ces protéines contenant le composant-3 sont isolées par chromatographie d'interactions hydrophobes. Les rendements d'extraction sont améliorés par optimisation de ce protocole. Le comportement physicochimique des fractions protéiques (protéose-peptones totales et leurs fractions hydrophobes) est étudié. L'électrophorèse en gel de polyacrylamide en milieu non dissociant (PAGE native) montre une plus faible mobilité du composant-3 des laits ovin et caprin par rapport à leur homologue du lait bovin. En milieu dissociant, en présence de dodécylsulfate de sodium et du 2-mercaptoéthanol (PAGE-SDS), les profils électrophorétiques des fractions hydrophobes ont en commun 2 bandes principales correspondant à des glycoprotéines de 18000 et $28000 \mathrm{Da}$. Ces polypeptides sont impliqués dans les agrégats de hauts poids moléculaires mis en évidence par chromatographie de perméation sur gel (colonnes de Séphacryl S200 et Superose 12). L'électrophorèse bidimensionnelle a montré une bonne similitude entre les fractions protéiques des laits des 3 espèces, concernant leurs points isoélectriques et leurs poids moléculaires. La composition chimique (acides aminés, glucides, phosphore) a montré également des analogies entre les fractions protéose-peptones des laits provenant des espèces laitières étudiées.
\end{abstract}

protéose-peptone / fraction hydrophobe / lait / brebis / chèvre

Summary - Isolation and characterization of proteose-peptone hydrophobic fractions of cow's, ewe's and goat's milk. The proteose-peptone extraction and purification procedure, largely utilized until now for cow's milk, was adapted for ewe's and goat's milk. The proteose-peptone fractions which contain component-3 were isolated by hydrophobic interaction chromatography. The extraction yield was increased by the improvement of the procedure. Physico-chemical behaviours of protein fractions (total proteose-peptone and their hydrophobic components) were studied. The native polyacrylamide gel electrophoresis (native PAGE) showed that ewe's and goat's component-3 migrations were lower than those of their cow's milk counterpart. The electrophoretic patterns of the hydrophobic fractions obtained in denaturating conditions with sodium dodecyl sulfate and 2mercaptoethanol (SDS-PAGE), had 2 major common bands corresponding to glycoproteins

\footnotetext{
*Adresse actuelle : ELAIS, 74 Achinon-Pireos Str 18547 Piraeus, Grèce
} 
of 18000 and $28000 \mathrm{Da}$. These polypeptides were implicated in the high molecular weight aggregates which were revealed by gel permeation chromatography (Sephacryl S200 and Superose 12 columns). Two-dimensional electrophoresis showed high similarity among the different protein fractions regarding their isoelectric points and molecular weights. The chemical composition (amino acids, carbohydrates, phosphorus) also showed analogies between the proteose-peptone fractions belonging to the 3 milk species.

proteose-peptone / hydrophobic fraction / cow milk / ewe milk / goat milk

\section{INTRODUCTION}

La fraction protéose-peptone du lait peut être divisée en 2 groupes distincts selon leurs origines. Le premier comprend des fragments protéiques provenant de l'hydrolyse des caséines par les protéases du lait. Ces constituants correspondent aux composants $\beta-C N-5 P, \beta-C N-4 P$ et $\beta-C N$ $1 \mathrm{P}$, dénommés précédemment et respectivement PP5, PP8(F) et PP8(S) (Andrews, 1979; Eigel et Keenan, 1979; Andrews et Alichanidis, 1983 et Eigel et al, 1984). Le second groupe est composé de protéines et polypeptides présents à l'état natif dans le lactosérum et dont le composant-3 (PP3) est le constituant majeur. Ce dernier se retrouve dans une fraction à caractère très hydrophobe dénommée FHPP (fraction hydrophobe des protéose-peptones) par Pâquet et al (1985). II est en outre caractérisé par des propriétés tensio-actives intéressantes (Shimizu et al, 1989), une thermorésistance élevée (Pearce, 1980) et un rôle important qu'il semble jouer dans l'inhibition de la lipolyse spontanée du lait (Anderson, 1981; Cartier et Chilliard, 1986 et Cartier et al, 1990).

Ces propriétés ont permis la mise en œuvre de techniques diverses d'isolement et de purification de ce composant. Les plus utilisées sont les relargages successifs au sulfate d'ammonium en partant d'un lait non chauffé ( $\mathrm{Ng}$ et al, 1970; Nejjar et al, 1990), la chromatographie d'interactions hydrophobes $(\mathrm{ClH})$ (Pâquet et al,
$1985)$ et enfin la chromatogrophie d'affinité sur concanavaline A (Kester et Brunner, 1982). Tout récemment, Kanno (1989a, b) isole une fraction analogue au PP3 qu'il dénomme lactophorine. Cette dernière est purifiée et fractionnée en 7 constituants par chromatographies d'échange d'ions et de perméation sur gel, alors que Girardet et al (1991) utilisent la chromatographie liquide haute-performance (FPLC) et l'électrophorèse bidimensionnelle pour la séparation et la caractérisation de fractions de protéose-peptones de lait bovin.

Les connaissances acquises à ce jour sur cette fraction protéique du lait ont été établies en majeure partie sur le lait de vache. La fraction analogue dans les laits d'autres espèces laitières n'a fait l'objet que de quelques investigations très limitées. À ce titre, seuls les travaux de Bezkorovainy et al (1976) sur le lait humain, de Ramos et al (1988) sur les laits de chèvre et brebis et enfin de Khatoon et Joshi $(1989 a, b)$ sur le lait de chèvre peuvent être relevés. De plus, ces quelques études se sont intéressées aux protéosepeptones totales. Le fractionnement de ces dernières n'a pu être réalisé que sur le lait humain.

Le présent travail s'est proposé d'isoler à partir des laits de 3 espèces laitières (bovine, ovine et caprine) des préparations de protéose-peptones et de leurs fractions hydrophobes contenant le composant-3. Ces fractions sont étudiées en comparant leurs comportements tant chromatographiques 
(interactions hydrophobes et perméation sur gel), électrophorétiques (en milieu dissociant et non dissociant, en focalisation isoélectrique et électrophorèse bidimensionnelle) que physico-chimiques (traitement thermique, précipitations acide et saline). Leur constitution chimique (acides aminés, glucides, azote et phosphore) a également été examinée.

\section{MATÉRIEL ET MÉTHODES}

\section{Préparation des protéose-peptones totales (PPT)}

Les protéose-peptones sont extraites de laits crus de mélange provenant de troupeaux de vaches (race Holstein), de brebis (race Lacaune) et de chèvres (race Alpine) de la région Est de la France (Lorraine). Pour l'isolement des PPT de lait de brebis et de chèvre, nous avons adapté le protocole d'Aschaffenburg (1946) utilisé essentiellement jusqu'à présent pour le lait bovin. Pour cela, nous avons testé une gamme de températures allant de 70 à $100^{\circ} \mathrm{C}$ pendant $30 \mathrm{~min}$ pour le traitement thermique et différentes concentrations en sulfate d'ammonium allant de 30 à $70 \%$ de saturation pour la précipitation des protéines dans le surnageant de thermocoagulation. Le précipité ainsi obtenu est, selon les cas, soit recueilli directement dans le tampon de départ de la chromatographie d'interactions hydrophobes, soit dissous dans un volume minimal d'eau puis dialysé, concentré, congelé et enfin lyophilisé.

\section{Préparation des fractions hydrophobes contenant le PP3}

Les PPT sont fractionnées par la chromatographie d'interactions hydrophobes $(\mathrm{ClH})$. Initialement, nous avons appliqué la méthode de Pâquet et Alais (1982), nous déposons $300 \mathrm{mg}$ de lyophilisat de PPT en solution dans $10 \mathrm{ml}$ de tampon (phosphate de sodium 0,01 mol. $.^{-1}, \mathrm{pH}$ 6,8 et sulfate d'ammonium 0,35 mol. $^{-1}$ ) au sommet de la colonne de Phényl-Sépharose CL-4B
(Pharmacia Fine Chemicals, Uppsala, Suède). Afin d'augmenter les rendements de purification des fractions protéose-peptones, nous avons optimisé ce protocole. Pour ce faire, le précipité issu du surnageant de thermocoagulation est récupéré dans un volume minimal de tampon de départ de la $\mathrm{ClH}$. Après une estimation de la quantité de protéines par la méthode de Lowry et al (1951), il est déposé à raison de 200-300 $\mathrm{mg}$ de protéines et fractionné sur une colonne $(2,6 \times 25 \mathrm{~cm})$ de Phényl-Sépharose $\mathrm{CL}-4 \mathrm{~B}$ avec un débit d'élution de $54 \mathrm{ml} / \mathrm{h}$.

\section{Caractérisations physico-chimiques et chimiques}

\section{Méthodes chromatographiques}

La FHPP issue de la $\mathrm{CIH}$ est dialysée contre le tampon (Tris- $\mathrm{HCl} 0,1 \mathrm{~mol}^{-1}{ }^{-1} ; \mathrm{NaCl} 0,5 \mathrm{~mol}^{-1}{ }^{-1}$; $\mathrm{pH} 6,8$ ) puis déposée à raison de $30 \mathrm{mg}$ de protéines sur une colonne $(2,5 \times 15 \mathrm{~cm})$ remplie de Séphacryl S200 (Pharmacia Fine Chemicals). $\mathrm{Ce}$ gel a une limite d'exclusion de $250000 \mathrm{Da}$. Les protéines sont éluées avec un débit de 18 $\mathrm{ml} / \mathrm{h}$. Avec le système FPLC (Pharmacia Fine Chemicals) cette même fraction est séparée par filtration sur une colonne Superose 12 HR 10/30 (Pharmacia Fine Chemicals). 0,5 mg de protéines sont injectées sur la colonne, équilibrée avec le tampon (Tris- $\mathrm{HCl} 0,05$ mol. $^{-1} ; \mathrm{NaCl}$ 0,15 mol..$\left.^{-1} ; \mathrm{pH} 8\right)$. L'élution a lieu avec un débit de $15 \mathrm{ml} / \mathrm{h}$.

\section{Méthodes électrophorétiques}

Elles sont réalisées sur gels de polyacrylamide (BDH, Poole, Grande-Bretagne). En milieu non dissociant (PAGE-native), la méthode de Hillier (1976) est adaptée à un système vertical 2001 (LKB, Bromma, Suède). En milieu dissociant, en présence de dodécylsulfate de sodium et de 2mercaptoéthanol (PAGE-SDS), la méthode en tampons discontinus de Laemmli et Favre (1973) est appliquée. Elle utilise un gel de séparation $(\mathrm{T}: 15,4 \% ; \mathrm{C}: 2,7 \%$ ) en tampon (Tris- $\mathrm{HCl}$ $\left.0,38 \mathrm{~mol}^{-1} \mathrm{I}^{-1} \mathrm{pH} 8,8\right)$ et un gel de concentration ( $T$ : $4,5 \% ; C: 2,7 \%$ ) en tampon (Tris- $\mathrm{HCl}$ 0,125 mol. $\left.^{-1}, \mathrm{pH} 6,8\right)$. Le coffret de protéines standard (Serva Fine Biochemicals, Heidelberg, 
RFA) contient : I'albumine sérique bovine (66 $000 \mathrm{Da})$, l'ovalbumine (45000 Da), la glycéraldéhyde-3-phosphodéshydrogénase (36 000 $\mathrm{Da})$, l'anhydrase carbonique (29000 Da), le trypsinogène (24000 Da), l'inhibiteur trypsique $(20100 \mathrm{Da})$ et l' $\alpha$-lactalbumine (14 $200 \mathrm{Da})$. La focalisation isoélectrique (FIE) a été réalisée selon la méthode optimisée par Sanogo et al (1990) en utilisant un gel ( $T$ : $5 \%$; $C$ : $3 \%$ ) contenant de l'urée 7 mol. $^{-1}$ et des Pharmalytes (LKB) $2,4 \%(v / v)$ couvrant chacun une zone de $\mathrm{pH}: 2,5-5,4-6$ et 3-10. L'électrophorèse bidimensionnelle couplant la FIE et la PAGE-SDS a été conduite selon la technique préconisée par Trieu-Cuot et Gripon (1981). La révélation des protéines est réalisée avec le Bleu de Coomassie R250 (Serva Fine Biochemicals), 0,1\% (p/v) en solution dans un mélange de méthanol $(50 \%, v / v)$ et d'acide trichloracétique (TCA $12 \%$, $\mathrm{p} / \mathrm{v})$. Les glycoprotéines sont mises en évidence par la technique de Kapitany et Zebrowski (1973) en utilisant l'acide périodique $(1 \%, p / v)$ et le réactif de Schiff.

\section{Méthodes chimiques}

L'azote est dosé par la technique du microKjeldahl (FIL-IDF 20, 1962). Les protéines sont estimées par la méthode colorimétrique de Lowry et al (1951) avec l'albumine sérique bovine comme protéine de référence. Le phosphore est dosé par la méthode FIL-IDF 33B (1982). La composition en acides aminés des protéines est déterminée par auto-analyseur Technicon NC2P après hydrolyse acide à $110^{\circ} \mathrm{C}$ pendant $24 \mathrm{~h}$, en présence d'acide chlorhydrique $6 \mathrm{~N}$. Les teneurs en glucides sont estimées par dosages colorimétriques décrits par Montreuil et Spik (1963), à savoir : les oses neutres selon la méthode à l'orcinol sulfurique utilisant un mélange de mannose et de galactose pour la gamme étalon; les hexosamines par la méthode d'Elson et Morgan (1933) avec des réactifs à l'acétyl-acétone et au paradiméthylaminobenzaldéhyde; enfin, les acides sialiques sont dosés par la méthode de Warren (1959) en faisant intervenir du métapériodate de sodium, de l'arsénite de sodium et de l'acide thiobarbiturique.

\section{RÉSULTATS}

\section{Isolement des protéose-peptones}

Les conditions optimales d'extraction des protéose-peptones sont similaires pour les 3 espèces laitières. Le traitement thermique à $95^{\circ} \mathrm{C}$ pendant 30 min dénature l'ensemble des caséines et des protéines lactosériques qui précipitent à $\mathrm{pH} 4,6$ (pour les laits de vache et de brebis) et à $\mathrm{pH} 4,2$ (pour celui de la chèvre). Le sulfate d'ammonium utilisé à $50 \%$ de saturation précipite la totalité des protéose-peptones contenues dans le surnageant de thermocoagulation. La précipitation au TCA 12\% $(p / v)$ aboutit à un résultat identique. Le taux de protéose-peptones obtenu avec ce protocole (fig 1) est en moyenne de $1 \mathrm{~g} / \mathrm{l}$ de lait, soit : 1,4 g/l pour le lait de brebis, 1 $\mathrm{g} / \mathrm{l}$ pour le lait de vache et $0,6 \mathrm{~g} / \mathrm{l}$ pour le lait de chèvre.

\section{Chromatographie d'interactions hydrophobes}

Dans le protocole d'obtention directe de la fraction hydrophobe à partir de protéosepeptones non lyophilisées (fig 1, voie 2), nous avons réalisé un gradient décroissant $\left(0,5-0 \mathrm{~mol}^{-1} \mathrm{I}^{-1}\right)$ en sulfate d'ammonium afin de déterminer la molarité nécessaire pour fractionner ces protéines. Ce gradient a permis d'établir, expérimentalement, qu'une concentration équivalente à 0,05 mol..$^{-1}$ en sulfate d'ammonium dans le tampon de départ de la $\mathrm{ClH}$ est suffisante pour retenir tout le composant-3 des protéose-peptones dans la matrice de PhénylSépharose. Le PP3 se retrouve entière- 


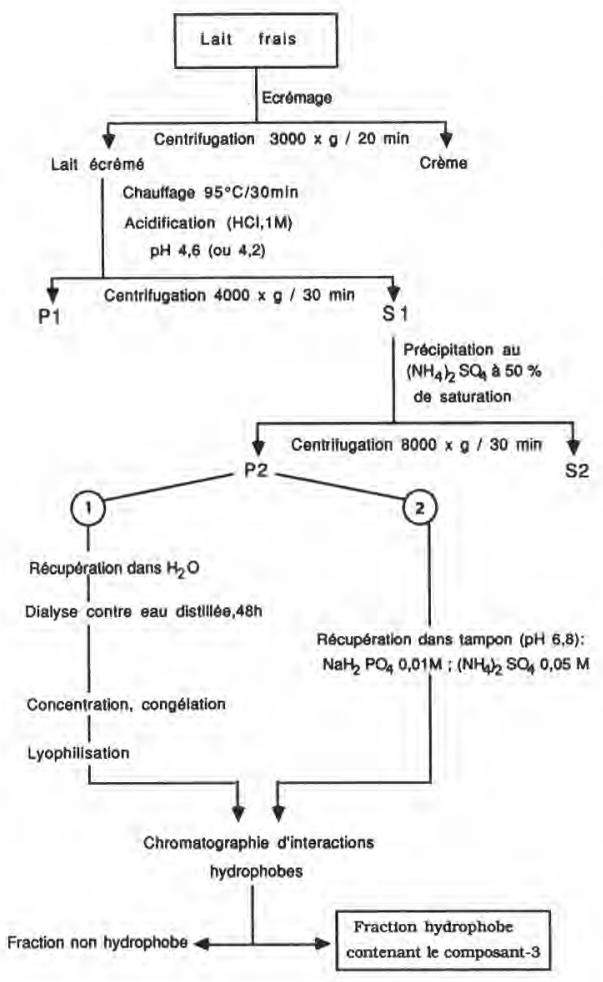

Fig 1. Protocole d'isolement de la fraction hydrophobe des protéose-peptones contenant le composant-3. 1: voie utilisée par Pâquet et Alais (1982). 2: nouvelle voie proposée. P1: précipité de protéines thermocoagulées (caséines et séroprotéines dénaturées). P2: précipité de protéose-peptones totales (PPT). S1: surnageant contenant les protéose-peptones, azote non protéique, lactose et sels minéraux. S2: surnageant contenant l'azote non protéique, lactose et sels. Procedure of isolation of proteose-peptone hy drophobic fractions containing component-3. 1: Method used by Pâquet and Alais (1982). 2: New method proposed. P1: Pellet of denatured proteins (caseins and major whey proteins). P2: Pellet of total proteose-peptone (TPP). S1: Supernatant containing proteose-peptone, non protein nitrogen, lactose and mineral salts. S2: Supernatant containing non protein nitrogen, lactose, and mineral salts. ment dans la FHPP (fig 2, fraction 2) et se sépare des autres composants : $\beta-\mathrm{CN}-5 \mathrm{P}$, $\beta-C N-4 P$ et $\beta-C N-1 P$ qui sont eux élués dans une fraction peu ou pas hydrophobe que nous appelons : fraction non hydrophobe des protéose-peptones (FNHPP) (fig 2, fraction 1). L'élution de FHPP est obtenue en substituant au sulfate d'ammonium $0,05 \mathrm{~mol}^{-\mathrm{I}^{-1}}$ une solution d'éthylène glycol à $50 \%(\mathrm{v} / \mathrm{v})$. Cette fraction obtenue en $3 \mathrm{~h}$ d'élution représente environ $30-40 \%$ des protéines totales éluées, soit $300-400 \mathrm{mg} / \mathrm{l}$ de lait frais. Les protéose-peptones des 3 types de laits fractionnées dans ces conditions donnent des résultats analogues et reproductibles.

\section{Chromatographie de perméation sur gel}

En chromatographie de perméation sur gel de Séphacryl S-200, les profils obtenus (fig 3 A) pour les FHPP des 3 types de laits sont similaires. Ils présentent 2 pics majeurs. Le premier correspond aux protéines éluées dans le volume d'exclusion de la colonne et représente $60-80 \%$ des protéines totales. Le second pic plus étalé regroupe des protéines et polypeptides de faible poids moléculaire. Le PP3 se retrouve dans le premier pic, aussi bien dans le lait de vache que dans les laits de brebis et de chèvre.

En FPLC sur colonne de Superose 12, les mêmes fractions sont séparées en 120 min en 4 pics majeurs avec des temps de rétention identiques chez les 3 espèces (fig $3 \mathrm{~B}$ ). Au bout de $25 \mathrm{~min}$, les fractions éluées contenues dans le volume d'exclusion de la colonne (pic 1) correspondent à des protéines de poids moléculaires (PM) élevés (250 000 à $450000 \mathrm{Da}$ ). Les fractions éluées au bout de 40-50 min (pic 2) ont des PM moyens (autour de 110000 $\mathrm{Da})$. Les fractions correspondant aux pics 3 et 4 renferment les composants ayant 


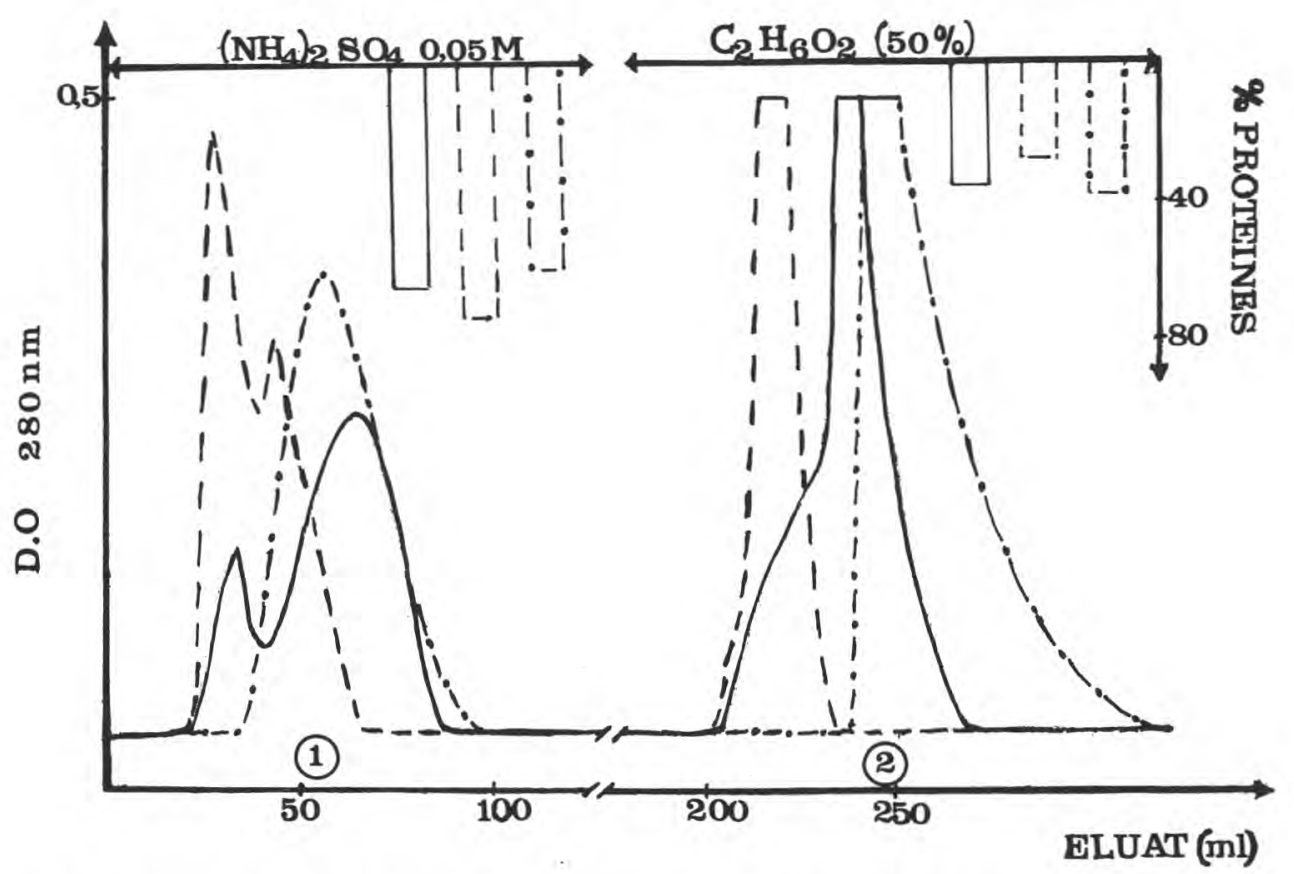

Fig 2. Chromatographie de protéose-peptones sur une colonne $(2,6 \times 25 \mathrm{~cm})$ de Phényl-Sepharose CL-4B; dépôt : $200 \mathrm{mg}$ de protéines, débit d'élution : $54 \mathrm{ml} / \mathrm{h}$. (-) lait de vache, $(--)$ lait de brebis, $(\rightarrow-)$ lait de chèvre. 1: fraction non hydrophobe des protéose-peptones (FNHPP), 2: fraction hydrophobe des protéose-peptones (FHPP).

Chromatography of proteose-peptone on a phenyl-Sepharose CL-4B column $(2.6 \times 25 \mathrm{~cm})$. Sample: $200 \mathrm{mg}$ proteins, flow rate: $54 \mathrm{ml} / \mathrm{h}$. (-) cow milk; (- -) ewe milk; (-o) goat milk. 1: Non hydrophobic fraction of proteose-peptone (NHFPP), 2: Hydrophobic fraction of proteose-peptone (HFPP).

les plus faibles PM (inférieurs à 30000 Da).

\section{Comportement électrophorétique des fractions protéiques}

En PAGE native, les profils des protéosepeptones (fig 4 A) des laits bovin, ovin et caprin présentent beaucoup de similitudes. Néanmoins, les protéose-peptones des laits de brebis et chèvre se distinguent par une mobilité électrophorétique plus faible de la bande correspondant au com- posant-3 (I) et se caractérisent par des profils moins complexes. Les deux bandes intenses correspondant à $\beta-\mathrm{CN}-5 \mathrm{P}$ (II) dans le lait de vache se retrouvent au même niveau chez la chèvre et la brebis, avec toutefois, une migration légèrement plus élevée dans ce dernier cas. Les FNHPP (piste 2) issues de la CIH représentées sur le même électrophorégramme (fig 4A) contiennent les composants correspondant à $\beta-\mathrm{CN}-5 \mathrm{P}, \quad \beta-\mathrm{CN}-4 \mathrm{P}$ et $\beta-C N-1 P$. Les FHPP (piste 3) sont constituées essentiellement du PP3. Les composants protéiques à plus fortes mobilités 

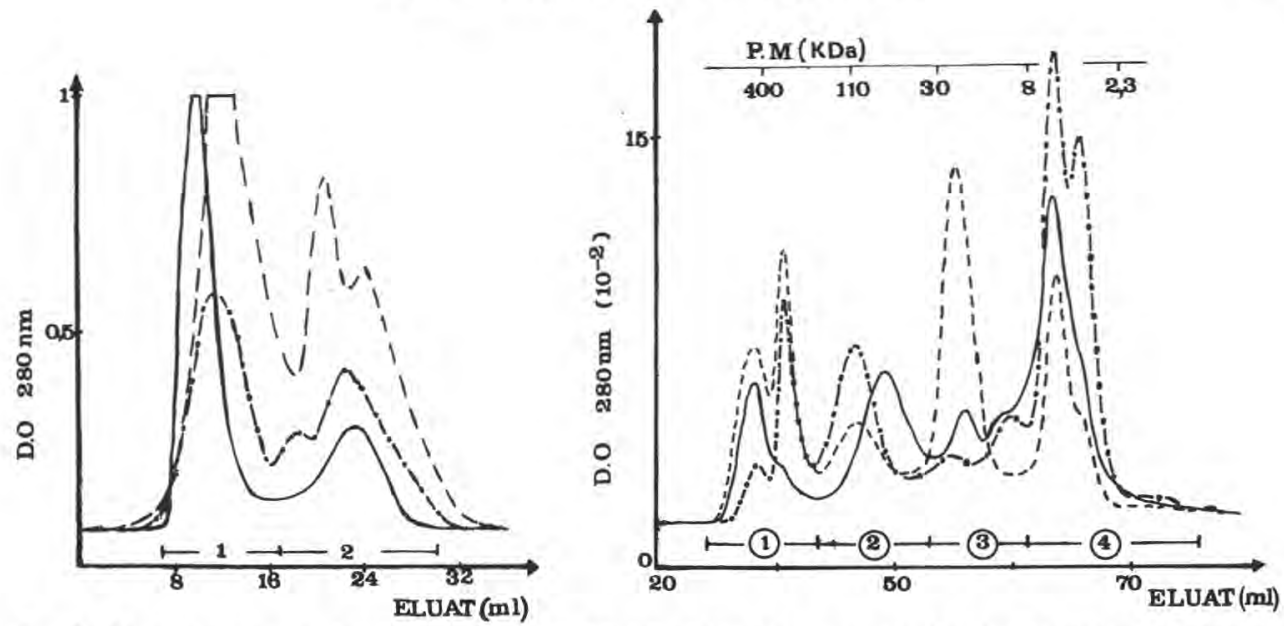

Fig 3. Chromatographie de perméation sur gel de la fraction hydrophobe des protéose-peptones (FHPP). a: colonne $(2,5 \times 15 \mathrm{~cm})$ de Séphacryl S200 équilibrée avec le tampon (Tris- $\mathrm{HCl} 0,1$ mol.1-1, $\mathrm{NaCl} 0,5$ mol. $\mathrm{I}^{-1}, \mathrm{pH} 6,8$ ); dépôt : $30 \mathrm{mg}$ de protéines, débit d'élution : $18 \mathrm{ml} / \mathrm{h}$. b: colonne de Superose $12 \mathrm{HR}\left(10 / 30\right.$ ) équilibrée avec le tampon (Tris- $\mathrm{HCl} 0,05$ mol. $\mathrm{I}^{-1}$, $\mathrm{NaCl} 0,15 \mathrm{~mol}^{. \mathrm{I}^{-1}} ; \mathrm{pH} 8$ ); injection $0,5 \mathrm{mg}$ de protéines, débit d'élution : $15 \mathrm{ml} / \mathrm{h}$. (-) lait de vache, $(--)$ lait de brebis; $(--)$ lait de chèvre.

Gel permeation chromatography of proteose-peptone hydrophobic fractions. A: Sephacryl-S200 column $(2.5 \times 15 \mathrm{~cm})$ equilibrated with Tris- $\mathrm{HCl} 0.1$ mol. $^{-1}, \mathrm{NaCl} 0.5 \mathrm{M} ; \mathrm{pH} 6.8$ buffer. Sample: $30 \mathrm{mg}$ proteins; flow rate: $18 \mathrm{ml} / \mathrm{h}$. B: Superose $12 \mathrm{HR}$ (10/30) column equilibrated with Tris- $\mathrm{HCl}$ $0.05 \mathrm{~mol}^{-\mathrm{t}^{-1}}, \mathrm{NaCl} 0.15 \mathrm{~mol}^{-\mathrm{I}^{-1}}$; $\mathrm{pH} 8$ buffer. Injection: $0.5 \mathrm{mg}$ proteins; flow rate: $15 \mathrm{ml} / \mathrm{h}$. (-) cow milk; $(--)$ ewe milk; (-) goat milk.

électrophorétiques ne représentent qu'une faible proportion dans cette fraction.

L'électrophorèse en PAGE-SDS des PPT (fig 4B) montre l'existence de 3 zones correspondant à des groupes de polypeptides dont les PM sont évalués à 12000 , 18000 et $28000 \mathrm{Da}$. La FNHPP du lait de vache migre en 2 bandes majeures de PM : 12000 et $18000 \mathrm{Da}$. La fraction analogue du lait de brebis migre en une bande assez large autour de $18000 \mathrm{Da}$, alors que celle du lait de chèvre se répartit en 3 bandes dont les PM sont évalués à 8000 , 14000 et $18000 \mathrm{Da}$. Les profils électrophorétiques des FHPP dans les mêmes conditions présentent une grande ressemblance surtout entre les laits de vache et de brebis. Les fractions issues de ces laits ont en commun des bandes majeures (18000 et $28000 \mathrm{Da}$ ) et des bandes mi- neures (14 000 et $16000 \mathrm{Da})$. La FHPP des laits de chèvre se distingue par un nombre plus élevé de bandes protéiques. Trois d'entre elles ont des PM proches de 15000,17000 et $20000 \mathrm{Da}$. Les 2 autres sont les bandes principales de 18000 et $28000 \mathrm{Da}$. Ces deux dernières sont communes aux FHPP des laits des 3 espèces. De plus, elles portent la coloration spécifique des glycoprotéines. Les composants $\beta-C N-5 P, \beta-C N-4 P$ et $\beta-C N-1 P$ ne semblent pas être de nature glycoprotéique; la FNHPP qui les contient n'est pas révélée par la coloration au réactif de Schiff.

L'analyse en PAGE-SDS des 4 fractions protéiques collectées en FPLC sur la colonne Superose 12 a montré que les 2 premières (pics 1 et 2) contiennent des polypeptides ayant un PM de $28000 \mathrm{Da}$, alors que les 2 autres (pics 3 et 4 ) renferment 

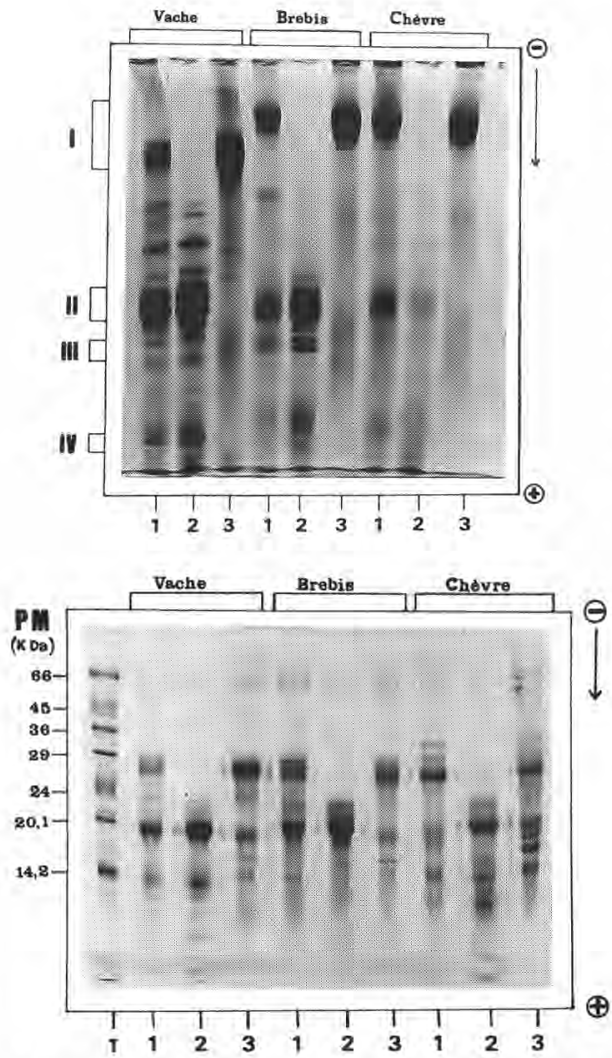

Fig 4. Électrophorèse en gel de polyacrylamide à $\mathrm{pH} 8,9$ des protéose-peptones et de leurs fractions protéiques issues de la chromatographie sur colonne de Phényl-Sépharose CL-4B. 1: protéose-peptones totales (PPT), 2: FNHPP, 3: FHPP. T: protéines standard. A: Images électrophorétiques en milieu non dissociant (PAGEnative). I: PP3; II: $\beta-C N-5 P$; III: $\beta-C N-1 P$; IV: $\beta$ $\mathrm{CN}-4 \mathrm{P}$. B: Images électrophorétiques en milieu dissociant en présence de dodécylsulfate de sodium et de 2-mercaptoéthanol (PAGE-SDS).

Polyacrylamide gel electrophoresis at $\mathrm{pH} 8.9$ of total proteose-peptone and fractions collected after chromatography on phenyl-Sepharose $C L-4 B$. 1: TPP, 2: NHFPP, 3: HFPP. T: standard proteins. A: patterns obtained in nondenaturing conditions (native-PAGE). I: PP3; II: $\beta-C N-5 P ; I I I: \beta-C N-1 P ; I V: \beta-C N-4 P$. B: patterns obtained in denaturing conditions, with sodium dodecyl sulfate and 2-mercaptoethanol (SDSPAGE). surtout des polypeptides à PM plus faibles (12 000 et $18000 \mathrm{Da}$ ); ceci indifféremment de l'origine du lait (résultats non présentés).

La FIE (fig 5) permet de dénombrer de nombreuses bandes protéiques ayant des points isoélectriques (PI) parfois très proches et distribués sur toute la gamme de $\mathrm{pH}$ (4-9). Sur le profil des PPT des 3 types de laits, nous dénombrons entre 40 et 50 bandes dont $2 / 3$ environ se situent entre $\mathrm{pH} 4$ et 5,5 . Les FNHPP sont constituées de composés acides (une vingtaine) dont les PI se situent entre 4 et 5,5. Le profil des FHPP se présente en une trentaine de bandes protéiques dont les $\mathrm{PI}$ se situent entre 4,5 et 9. La FHPP du lait de chèvre se caractérise par une proportion plus grande de composés à PI basiques. Enfin, le dénombrement des bandes protéiques migrant aux mêmes niveaux per-

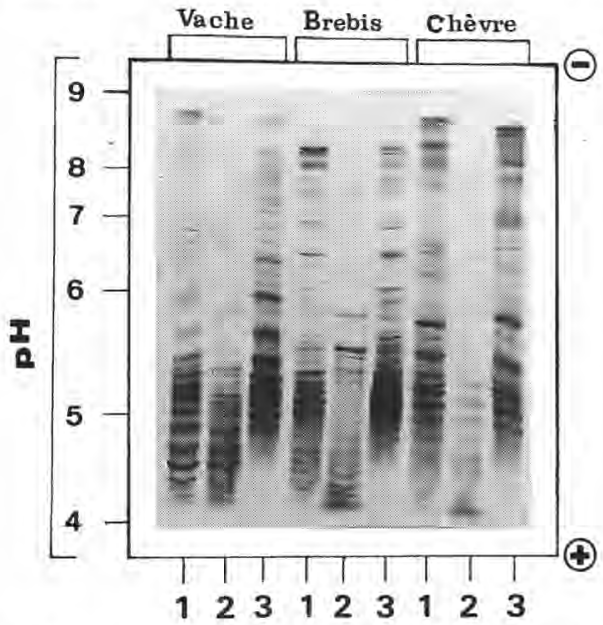

Fig 5. Focalisation isoélectrique (FIE) entre $\mathrm{pH}$ 4 et 9 des PPT et de leurs fractions protéiques issues de la chromatographie sur colonne de Phényl-Sépharose CL-4B. 1: PPT; 2: FNHPP; 3: FHPP.

Isoelectrofocusing (IEF) in a pH range of 4-9 of TPP and fractions collected after chromatography on phenyl-Sepharose CL-4B. 1: TPP; 2: NHFPP; 3: HFPP. 
met de constater qu'environ la moitié des composants de la fraction protéosepeptones des laits de brebis et de chèvre ont des PI identiques.

Les cartes bidimensionnelles des PPT (fig 6A, B et C) montrent une assez bonne concordance entre les laits des 3 espèces concernant le nombre et la position des spots correspondant aux polypeptides de 12000,18000 et $28000 \mathrm{Da}$. La bande protéique de $28000 \mathrm{Da}$ se subdivise en une dizaine de spots de PI différents allant de 4,5 à 7 . D'autres spots (de 2 à 5 selon
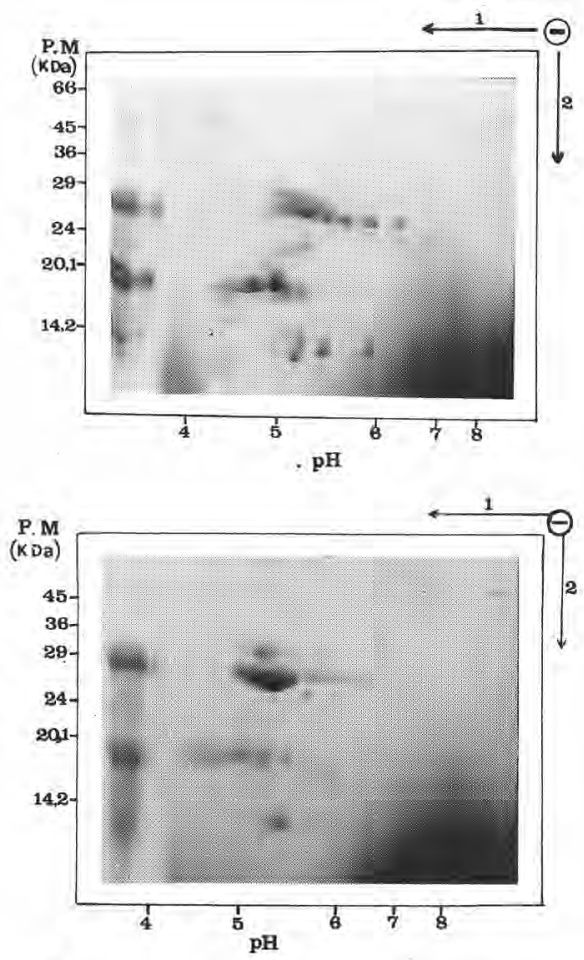

l'origines des PPT) sont identifiables sur la même zone de $\mathrm{pH}$ et auraient des PM légèrement plus élevés (autour de 30000 Da). La bande protéique de $18000 \mathrm{Da}$ se décompose en 5 spots environ ayant des $\mathrm{Pl}$ acides compris entre 4 et 5,5 (dans les laits des vache et de brebis) et entre 4,5 et 7 (dans le lait de chèvre). Enfin , nous dénombrons 4 spots pour la bande protéique de $12000 \mathrm{Da}$. Dans le cas de FHPP dont le comportement en électrophorèse bidimensionnelle est résumé par la cartographie (fig 6D), nous retrouvons la grande
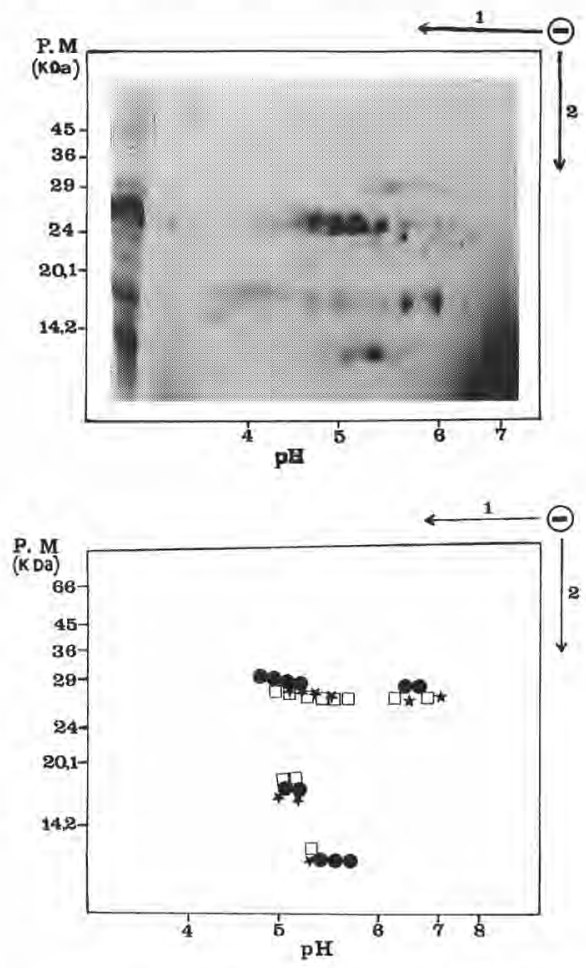

Fig 6. Électrophorèse bidimensionnelle des protéose-peptones et de leurs fractions hydrophobes. 1: FIE; 2: PAGE-SDS. A: PPT du lait de vache; B: PPT du lait de brebis; C: PPT du lait de chèvre; D: cartographie des FHPP, (口) spots de FHPP du lait de vache, (*) spots de FHPP du lait de brebis, ( spots de FHPP du lait de chèvre.

Bidimensional electrophoresis of total proteose-peptone and their hydrophobic fractions. 1: IEF; 2: SDS-PAGE. A: TPP of cow milk; B: TPP of ewe milk; C: TPP of goat milk. D: cartography of HFPP; (घ) HFPP components of cow milk; (*) HFPP components of ewe milk; (ब) HFPP components of goat milk. 
partie des spots ayant des PM de 28000 $\mathrm{Da}, 2$ spots à $18000 \mathrm{Da}$ et de $\mathrm{PI}$ proches de 5 ainsi que 1 ou 3 spots à $12000 \mathrm{Da}$.

\section{Analyses chimiques}

La comparaison des aminogrammes (tableau I) montre que les teneurs en acides aminés sont assez proches entre les fractions PPT des laits des 3 espèces. Toutefois, nous pouvons relever que les PPT du lait de vache ont un taux plus élevé en sérine; les PPT du lait de brebis se distinguent par un taux plus important en acide aspartique, glycine et méthionine; enfin, les PPT du lait de chèvre possèdent une teneur plus élevée en proline. Les FHPP des laits de brebis et de chèvre ont des teneurs plus importantes en. lysine et plus faibles en sérine que la fraction homologue du lait de vache.

Le dosage colorimétrique des glycoprotéines (tableau II) permet d'estimer cette fraction à environ $7 \%$ dans les PPT du lait de vache et $11 \%$ dans celles des laits de brebis et de chèvre. Les FHPP des laits de vache et de brebis renferment respectivement 9,5 et $10 \%$ de glucides contre $12,2 \%$ pour la même fraction issue du lait de chèvre. Plus de la moitié de la partie glucidique des FHPP est constituée d'oses neutres. Les fractions protéiques du lait de chèvre sont celles qui renferment les teneurs les plus élevées en oses neutres, hexosamines et acides sialiques. Les teneurs en phosphore total des différentes fractions (PPT et FHPP) semblent plus faibles dans le cas des fractions des laits de brebis et de chèvre.

Tableau I. Composition en acides aminés (exprimée en mol \%) des protéose-peptomes et de leurs fractions hydrophobes. 1 : PPT; 2 : FHPP; $T r$ : traces.

Amino-acid composition (mol \%) of total proteose-peptone and their hydrophobic fractions. 1: TPP; 2:HFPP; Tr: trace amount.

\begin{tabular}{|c|c|c|c|c|c|c|}
\hline \multirow{2}{*}{$\begin{array}{c}\text { Espèce } \\
A A\end{array}$} & \multicolumn{2}{|c|}{ Vache } & \multicolumn{2}{|c|}{ Brebis } & \multicolumn{2}{|c|}{ Chèvre } \\
\hline & 1 & 2 & 1 & 2 & 1 & 2 \\
\hline Asp & 8,0 & 9,2 & 12,4 & 11,6 & 7,8 & 9,4 \\
\hline Thr & 8,4 & 7,7 & 7,3 & 7,8 & 8,2 & 7,9 \\
\hline Ser & 9,7 & 8,5 & 5,5 & 4,8 & 5,8 & 5,5 \\
\hline Glu & 17,2 & 15,4 & 9,2 & 6,3 & 18,7 & 15,6 \\
\hline Pro & 8,6 & 7,2 & 8,6 & 8,5 & 12,9 & 9,5 \\
\hline Gly & 3,1 & 3,6 & 6,4 & 7,2 & 2,7 & 3,9 \\
\hline Ala & 4,8 & 5,1 & 7,2 & 8,7 & 5,2 & 5,4 \\
\hline 1/2 Cys & $\operatorname{Tr}$ & 0,8 & 1,2 & 0,8 & $\mathrm{Tr}$ & 0,6 \\
\hline Val & 6,2 & 5,7 & 7,3 & 6,5 & 7,8 & 5,8 \\
\hline Met & 0,9 & 1,3 & 2,4 & 3,2 & $\mathrm{Tr}$ & 0,5 \\
\hline Ile & 5,5 & 5,9 & 6,6 & 4,4 & 5,9 & 5,7 \\
\hline Leu & 10,2 & 10,8 & 9,8 & 12,4 & 9,8 & 10,6 \\
\hline Tyr & 1,9 & 1,6 & 0,6 & 0,8 & 1,1 & 1,4 \\
\hline Phe & 3,6 & 3,4 & 3,8 & 2,9 & 3,2 & 2,6 \\
\hline Lys & 6,8 & 7,5 & 7,6 & 9,6 & 6,8 & 10,5 \\
\hline His & 2,5 & 3,5 & 2,4 & 2,5 & 1,9 & 2,4 \\
\hline Arg & 2,5 & 2,6 & 1,5 & 1,9 & 1,9 & 2,3 \\
\hline
\end{tabular}


Tableau II. Teneurs (mg/100 mg de protéines) en hexoses, hexosamines, acides sialiques et phosphore des protéose-peptones et de leurs fractions hydrophobes. 1 : PPT; 2 : FHPP.

Content (mg/100 mg protein) in hexose, hexosamine, sialic acid and phosphorus of total proteosepeptone and their hydrophobic fractions. 1: TPP; 2: HFPP.

\begin{tabular}{lllllll}
\hline Espèce & \multicolumn{2}{c}{ Vache } & \multicolumn{2}{c}{ Brebis } & \multicolumn{2}{c}{ Chèvre } \\
& 1 & 2 & 1 & 2 & 1 & 2 \\
\hline Oses neutres & 3,6 & 5,4 & 4,8 & 5,9 & 6,4 & 6,1 \\
Glucosamines & 1,8 & 2 & 1,4 & 2,7 & 2,9 & 3,4 \\
Acides sialiques & 1,5 & 2,13 & 1,3 & 1,9 & 1,6 & 2,7 \\
Phosphore total & 0,82 & 1,04 & 0,8 & 0,68 & 0,38 & 0,35 \\
\hline
\end{tabular}

\section{DISCUSSION}

La précipitation des protéose-peptones dans le surnageant de thermocoagulation conduit à des profils électrophorétiques similaires en utilisant le sulfate d'ammonium à $50 \%$ de saturation ou le TCA $12 \%(\mathrm{p} / \mathrm{v})$. Ramos et al (1988) ont abouti à la même conclusion en précipitant ces protéines au sulfate d'ammonium à saturation ou au TCA $12,5 \%(p / v)$. Néanmoins, nous avons préféré pour la suite de nos travaux l'utilisation du sel pour les conditions moins dénaturantes qu'il permet en plus de son intérêt en $\mathrm{ClH}$ dans l'augmentation de la force ionique du milieu.

La voie que nous avons introduite dans le protocole d'isolement des FHPP (fig 1 , voie 2) permet d'éviter les étapes de dialyse, concentration et lyophilisation. Ces étapes, selon les essais comparatifs que nous avons effectués, peuvent entrainer jusqu'à $25 \%$ de pertes en protéines. Ceci est loin d'être négligeable compte tenu des faibles quantités de FHPP $(300 \mathrm{mg} / \mathrm{l}$ de lait) obtenues à la fin du processus d'isolement.

L'analyse en PAGE native des PPT et des FHPP des laits provenant des 3 espèces considérées montre qu'il existe une grande ressemblance entre les profils de ces fractions, à l'exception de la mobilité électrophorétique plus élevée du PP3 du lait de vache par rapport à ses homologues ovin et caprin. Cette différence de mobilité se trouve confortée par les teneurs plus élevées en lysine, dans les FHPP des laits de brebis et de chèvre, donnant à ces dernières autant de charges positives en plus à $\mathrm{pH} 8,9$. Cette particularité du comportement des PP3 est en voie d'être utilisée par notre laboratoire pour la mise au point d'une méthode rapide de détection de l'adultération du lait de chèvre ou de brebis par le lait de vache.

En PAGE-SDS, les PPT, quelles que soient leurs origines, se répartissent en 3 groupes majeurs de polypeptides de 12000,18000 et $28000 \mathrm{Da}$. Des résultats similaires sont rapportés par Khatoon et Joshi (1987a) et par Ramos et al (1988). Quant aux FHPP, elles se composent de 2 groupes de polypeptides communs aux 3 types de laits et dont les PM sont estimés à 18000 et $28000 \mathrm{Da}$. Étant donné l'analogie apparente dans la constitution des FHPP des laits des 3 espèces étudiées, un rapprochement entre la fraction soluble des glycoprotéines de la membrane des globules gras et le PP3 est à envisager dans les laits de brebis et de chèvre. Ce rapprochement a déjà été effectué dans le lait bovin (Kester et Brunner, 1982; Nejjar et al, 1986 et Kanno, 1989a). Ces travaux concluent à une ideritité antigénique entre ces fractions et un comportement électro- 
phorétique similaire en PAGE-SDS (existence de 2 bandes glycoprotéiques de PM proches de 18000 et $28000 \mathrm{Da}$ ).

La focalisation isoélectrique a montré que les protéose-peptones sont constituées d'environ une quarantaine de groupes protéiques de PI différents et ce, indifféremment de l'espèce considérée. En adaptant la technique de FIE aux protéose-peptones du lait de vache, Kasper et Brunner (1978) séparent 23 constituants dont les PI sont compris entre 3,8 et 5,3; Pâquet et Alais (1982) dénombrent 30 bandes entre $\mathrm{pH} 4$ et 5,5 . Le nombre et la proximité des composés à $\mathrm{PI}$ très proches rend difficile l'utilisation de techniques d'échange d'ions pour la séparation de ces protéines (Andrews et Alichanidis, 1983). Toutefois, les protéose-peptones du lait de chèvre pourraient être fractionnées avec ce type de chromatographie, car possédant de nombreux composés basiques.

Pour les 3 types de lait (bovin, ovin et caprin), les protéose-peptones et leurs fractions hydrophobes présentent un comportement commun quand elles sont séparées par électrophorèse bidimensionnelle. Les bandes majeures de polypeptides de 12000,18000 et $28000 \mathrm{Da}$ se répartissent respectivement en 4, 5 et 10 composés ayant des PI compris entre 4 et 7 . Le PP3 se retrouve en majorité dans ce dernier groupe de constituants ( $P M=28000$ $\mathrm{Da})$, alors que les composants $\beta-\mathrm{CN}-5 \mathrm{P}$, $\beta-C N-4 P$ et $\beta-C N-1 P$ sont représentés dans le second groupe $(P M=18000 \mathrm{Da})$ par des spots à PI acides (de 4 à 5,5 ). L'ensemble de ces résultats concorde avec ceux obtenus par Girardet et al (1991) sur des fractions analogues de lait bovin séparées par FPLC.

Dans les conditions de la chromatographie de perméation sur gel (colonnes de Séphacryl S200 et de Superose 12), les polypeptides entrant dans la constitution du PP3 ont tendance à s'agréger pour former des polymères de hauts poids moléculaires (> $250000 \mathrm{Da}$ ). Ceci est observé dans les laits des 3 espèces laitières. Un comportement similaire est signalé pour le PP3 du lait bovin par $\mathrm{Ng}$ et al (1970). Pâquet et Alais (1982) et Pâquet et al (1985). De plus, les fractions collectées en FPLC sur Superose 12 ont révélé en PAGE-SDS que ce sont surtout les polypeptides de $28000 \mathrm{Da}$ qui sont impliqués dans cette agrégation.

La composition chimique des fractions étudiées, même si elle conforte les analogies observées jusque-là, peut justifier certaines différences de comportement men*. . aées plus haut. Dans l'ensemble, les teneurs en acides aminés des PPT et des FHPP du lait de vache concordent avec celles rapportées par de nombreux auteurs, notamment $\mathrm{Ng}$ et al (1970), Kanno et al (1975), Kester et Brunner (1982) et Pâquet et al (1988). Pour les fractions de laits de brebis et de chèvre, l'absence d'aminogramme dans les travaux rapportés dans ce domaine ne permet pas la comparaison avec nos résultats. Les teneurs en fractions glycoprotéiques des protéose-peptones de lait bovin $(7 \%)$, ovin (11\%) et caprin $(11 \%)$ observées sont plus élevées que celles obtenues par Ramos et al (1988) qui sont respectivement de 4, 7,3 et $6,4 \%$. II y a une variation, selon les auteurs, dans les taux de glycoprotéines exprimés pour la même fraction protéique. Ainsi, pour les protéose-peptones du lait de vache, la teneur en glucides totaux est estimée à 10\% par Bezkorovainy et al (1976) et Kester et Brunner (1982); alors que, Kanno et Yamauchi (1979) et Khatoon et Joshi (1989a) donnent des taux voisins de $6 \%$. Ceci pourrait s'expliquer par des différences dans les modes de préparation des fractions (laits chauffés ou non, précipitation acide ou saline) ainsi que dans les méthodes de dosage em- 
ployées (colorimétriques, chromatographiques ou autres).

Les ressemblances relevées dans la composition et les propriétés physicochimiques des fractions hydrophobes étudiées peuvent impliquer une analogie de fonction entre ces composants. Dans cette hypothèse, le constituant analogue du PP3 bovin contenu dans les laits de brebis et de chèvre jouerait un rôle d'effecteur dans la régulation de la lipolyse spontanée. D'autant qu'un effet d'inhibition important de la lipolyse dans le lait de chèvre par des préparations enrichies en protéosepeptones de ce même lait a été observé (Chilliard et al, 1984). Bien que cela n'ait pas été établi, il est probable, comme dans le cas du lait de vache (Cartier et al, 1990), que cet effet soit inhérent au PP3.

\section{CONCLUSION}

II ressort, au vu des résultats obtenus, qu'il existe une grande similitude entre les fractions de protéose-peptones des laits de vache, de brebis et de chèvre. Toutefois, les fractions issues de ces 2 derniers laits présentent entre elles encore plus de caractéristiques communes. Elles se distinguent particulièrement par rapport à leur homologue bovin par une plus faible mobilité électrophorétique du composant I (PP3) en PAGE native.

Pour les 3 espèces laitières étudiées, le PP3 est le constituant majeur de la fraction hydrophobe des protéose-peptones. Nos travaux montrent le caractère hétérogène de ce composant qui se présente dans les 3 types de laits comme un complexe phosphoglycoprotéique migrant en une bande diffuse en PAGE native et en 3 bandes majeures ( 12000,18000 et $28000 \mathrm{Da}$ ) en PAGE-SDS. De plus, en chromatographie de perméation sur gel, le PP3 s'agrège pour former des hétéroprotéines de haut poids moléculaire. Quant à l'origine de ce composant qui reste encore à élucider, les travaux réalisés sur le lait bovin ont montré qu'il proviendrait des protéines de structure de la membrane des globules gras. Cette hypothèse mérite d'être vérifiée sur les laits de brebis et de chèvre, eu égard aux caractéristiques communes relevées entre les FHPP étudiées.

\section{REMERCIEMENTS}

Nous tenons à remercier D Pâquet pour avoir initié et entamé avec nous cette étude ainsi que $M$ Prud'homme pour son assistance technique.

Ce travail a reçu une aide financière de la Région Lorraine.

\section{RÉFÉRENCES}

Anderson M (1981) Inhibition of lipolysis in bovine milk by proteose-peptone. J Dairy Res 48, 247-252

Andrews AT (1979) The formation and structure of some proteose-peptone components. $J$ Dairy Res 46, 215-218

Andrews AT, Alichanidis E (1983) Proteolysis of caseins and the proteose-peptone fraction of milk. J Dairy Res 50, 275-290

Aschaffenburg R (1946) Surface activity and proteins of milk. J Dairy Res 14, 316-329

Bezkorovainy A, Nichols JH, Sly. DA (1976) Proteose-peptone fractions of human and bovine milk. Int J Biochem 7, 639-645

Cartier P, Chilliard Y (1986) Effects of different skim milk fractions on activity of cow milk purified lipoprotein lipase. J Dairy Sci 69, 951955

Cartier P, Chilliard Y, Pâquet D (1990) Inhibition and activating effects of skim milks and proteose-peptone fractions on spontaneous lipolysis and purified lipoprotein lipase activity in bovine milk. J Dairy Sci 73, 1178-1186 
Chilliard Y, Selselet-Attou G, Bas P, MorandFehr P (1984) Characteristics of lipolytic system in goat milk. J Dairy Sci 67, 2216-2223

Eigel WN, Keenan TW (1979) Identification of proteose-peptone component 8-slow as a plasmin-derived fragment of bovine $\beta$ casein. Int J Biochem 10, 529-535

Eigel WN, Butler JE, Ernstrom CA, Farrel Jr HM, Harwalkar VR, Jenness R, McL Whitney R (1984) Nomenclature of proteins of cow's milk: fifth revision. J Dairy Sci 67,1599 1631

Elson LA, Morgan WTJ (1933) Cités par Montreuil J et Spik G (1963)

FIL-IDF standard 20 (1962) Determination of the total nitrogen content of milk by the Kjeldahl method.

FIL-IDF standard 33 B (1982) Determination of the total phosphorus content.

Girardet JM, Mati A, Sanogo T, Etienne L, Linden $G$ (1991) Fast protein liquid chromatography purification of hydrophobic fraction of bovine milk proteose-peptone and characterization by bidimensional electrophoresis. J Dairy Res 58, 85-98

Hillier RM (1976) The quantitative measurement of whey proteins using polyacrylamide gel electrophoresis. J Dairy Res 43, 259-265

Kanno C (1989a) Purification and separation of multiple forms of lactophorin from bovine milk whey and their immunological and electrophoretic properties. J Dairy Sci 72, 883891

Kanno C (1989b) Characterization of multiple forms of lactophorin isolated from bovine milk whey. J Dairy Sci 72, 1732-1739

Kanno C, Yamauchi K (1979) Relationship of soluble glycoprotein of milk fat globule membrane to component- $3,-5$ and 8 fractions of proteose-peptone. Agric Biol Chem 43, 2105-2113

Kanno C, Shimizu M, Yamauchi K (1975) Isolation and physico-chemical properties of a soluble glycoprotein fraction of milk fat globule membrane. Agric Biol Chem 39, 1835-1842

Kapitany RA, Zebrowski EJ (1973) A high resolution PAS stain for polyacrylamide gel electrophoresis. Anal Biochem 56, 361-369

Kasper GA, Brunner JR (1978) Electrophoretic characterization of the proteose-peptone fraction of cow's milk. J Dairy Sci (Suppl 1) $61,112-113$

Kester JJ, Brunner JR (1982) Milk fat-globule membrane as possible origin of proteosepeptone glycoproteins. J Dairy Sci 65, 22412252

Khatoon JA, Joshi VK (1989a) Physico-chemical characteristics of the proteose-peptone fraction from goat milk. I. Gel electrophoretic and filtration pattern. Milchwissenschaft 42, 236237

Khatoon JA, Joshi VK (1989b) Physico-chemical characteristics of the proteose-peptone fraction from goat milk. II. Amino acid composition hexose and hexosamine contents. Milchwissenschaft 42, 280-281

Laemmli UK, Favre H (1973) Maturation of the head of bacteriophage T4. I. DNA packaging events. J Mol Biol 80, 575-599

Lowry OH, Rosebrough NJ, Farr AL, Randall RJ (1951) Protein measurement with the Colin phenol reagent. J Biol Chem 193, 265-275

Montreuil J, Spik G (1963) Microdosage des glucides totaux. Fascicule 1. Méthodes colorimétriques de dosage des glucides totaux. Faculté des Sciences de Lille.

Nejjar Y, Pâquet D, Godbillon G, Le Deaut JY (1986) Immunological relationship between the hydrophobic fraction of proteose-peptone and the milk fat globule membrane of bovine milk. Int J Biochem 18, 893-900

Nejjar Y, Pâquet D, Aubert F, Linden G (1990) The PP3 component of the proteosepeptone. Extraction from unheated skim milk. Milchwissenschaft 45, 84-87

Ng WC, Brunner JR, Rhee KC (1970) Proteosepeptone fraction of bovine milk: lacteum serum component 3 , a whey glycoprotein. $J$ Dairy Sci 53, 987-1007

Pâquet D, Alais C (1982) Purification et quelques caractéristiques moléculaires du "composant 3 " des protéose-peptones. Lait $62,338-349$

Pãquet D, Nejjar Y, Alais C (1985) Electrophoretic and chromatographic behavior of the proteose-peptone fraction of cow's milk. Milchwissenschaft 40, 200-203

Pãquet D, Nejjar Y, Linden G (1988) Study of a hydrophobic protein fraction isolated from 
milk proteose-peptone. J Dairy Sci 71, 14641471

Pearce RJ (1980) Heat stable components in the Aschaffenburg and Drewry total albumin fraction from bovine milk. $N Z J$ Dairy Sci Technol 15, 13-22

Ramos M, Sanchez RM, Olano A, Sanz J, Martinez-Castro I (1988) Comparative studies on "acid-stable, heat-stable polypeptides" of ovine, caprine and bovine milks. Z Lebensm Unters Forsch 186, 22-24

Sanogo T, Pâquet D, Aubert F, Linden G (1990) Proteolysis of $\alpha s-1$ casein by papain in a complex environment. Influence of ionic strength on the reaction products. J Food Sci $55,796-800$

Shimizu M, Yamauchi K, Saito M (1989) Emulsifying properties of the proteose-peptone fraction obtained from milk. Milchwissenschaft 44, 497-500

Trieu-Cuot P, Gripon JC (1981) Electrofocusing and two-dimensional electrophoresis of bovine caseins. J Dairy Res 48, 303-310

Warren L (1959) cité par Montreuil J et Spik G (1963). 